\title{
Electromembrane Extraction and Mass Spectrometry for Liver Organoid Drug Metabolism Studies
}

\author{
Frøydis Sved Skottvoll, Frederik André Hansen, Sean Harrison, Ida Sneis Boger, Ago Mrsa, \\ Magnus Saed Restan, Matthias Stein, Elsa Lundanes, Stig Pedersen-Bjergaard, Aleksandra Aizenshtadt, \\ Stefan Krauss, Gareth Sullivan, Inger Lise Bogen, and Steven Ray Wilson*
}

Cite This: Anal. Chem. 2021, 93, 3576-3585

Read Online

ACCESS | 네 Metrics \& More | 回 Article Recommendations | sl Supporting Information

ABSTRACT: Liver organoids are emerging tools for precision drug development and toxicity screening. We demonstrate that electromembrane extraction (EME) based on electrophoresis across an oil membrane is suited for segregating selected organoid-derived drug metabolites prior to mass spectrometry (MS)-based measurements. EME allowed drugs and drug metabolites to be separated from cell medium components (albumin, etc.) that could interfere with subsequent measurements. Multiwell EME (parallelEME) holding $100 \mu \mathrm{L}$ solutions allowed for simple and repeatable monitoring of heroin phase I metabolism kinetics. Organoid parallel-EME extracts were compatible with ultrahigh-performance liquid chromatography (UHPLC) used to separate the analytes prior to detection. Taken together, liver organoids are well-matched with EME followed by MS-based measurements.

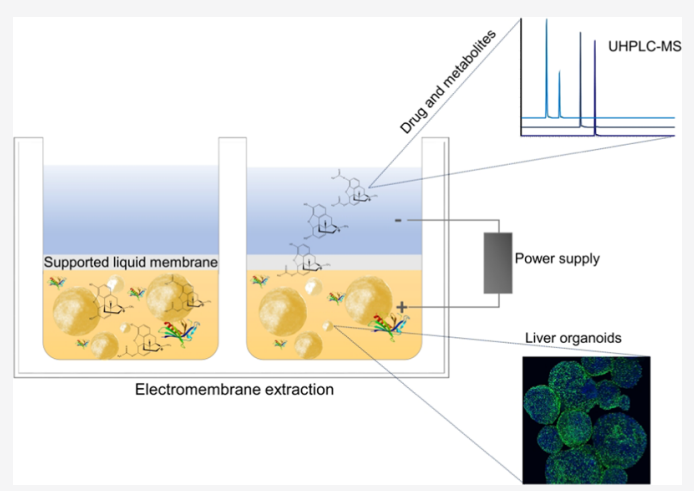

\section{INTRODUCTION}

The process of drug development is known to be timeconsuming and bear financial uncertainties. ${ }^{1,2}$ It is estimated that from 5000 to 10000 new molecular entities, only one new drug will enter the market. ${ }^{3}$ The advancement of this one drug from concept to market takes approximately 15 years and a cost of over $\$ 1$ billion, as well as the use of human resources, research skills, and technological expertise. ${ }^{3}$ As the majority of drug candidates are rejected late in the process and during clinical trials, ${ }^{3}$ one approach to reducing the assets put into the drug development may be to reject possible drug candidates early in the development process, i.e., during preclinical testing. This may be done by developing or utilizing in vitro models that adequately recapitulate the human in vivo response.

Organoids are three-dimensional tissue models derived from primary tissues, embryonic stem cells, or induced pluripotent stem cells (iPSC). ${ }^{4-6}$ These "mini" organs are emerging tools for studying human development and disease, serving as alternatives to cell cultures and animal models in drug development. ${ }^{7,8}$ A wide variety of organoids are being developed and studied, e.g., brain, heart, tumor tissue, and liver. $^{9-12}$ Liver organoids can be valuable models for studying drug metabolism and toxicity ${ }^{13}$ (Figure 1A), perhaps even in a personalized fashion, as organoids can be derived from the cells of a patient. ${ }^{14,15}$

Drug metabolism is a significant determinant of drug clearance and an indirect determinant of the clinical efficacy and toxicity of drugs. ${ }^{16}$ Thus, the mapping of the biotransformation pathway of drugs is crucial in the early part of the drug development process. ${ }^{17}$ Clinical studies of xenobiotics in humans are subjected to constraints concerning ethical aspects. Several in vitro model systems have been developed to recapitulate human functions from the molecular level to the cellular, tissue, organ, or whole organism level. The most commonly used in vitro models for drug metabolism studies include subcellular fractions, e.g., human liver microsomes (HLMs), S-9 fractions, and human hepatocytes. However, current in vitro models has some disadvantages. For example, HLMs do not represent a complete course of metabolism as they lack soluble phase II enzymes. ${ }^{16}$ Additionally, higher biotransformation rates are obtained in HLMs compared to humans, most likely because of the enriched enzyme concentrations and the absence of competing enzymes. ${ }^{17}$ Also, animal models can have shortcomings and have frequently been shown to lead to wrong predictions of drug interaction and toxicity in humans. ${ }^{18}$

For both in vitro and in vivo models, drug metabolism studies are very often performed utilizing liquid chromatography-mass spectrometry (LC-MS). Essentially, the mass spectrometer (MS) can measure the drugs and their

Received: December 4, 2020

Accepted: January 25, 2021

Published: February 3, 2021 
A

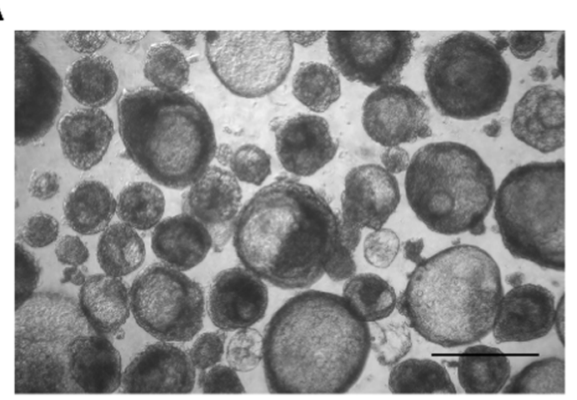

B

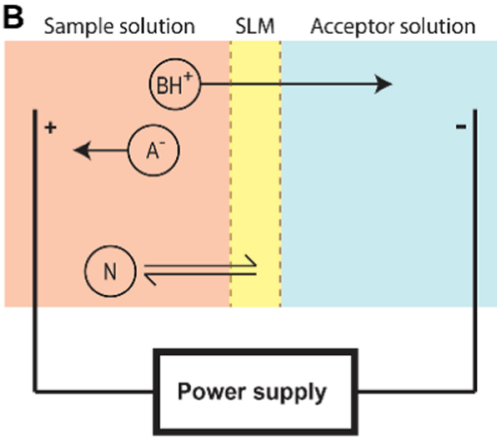

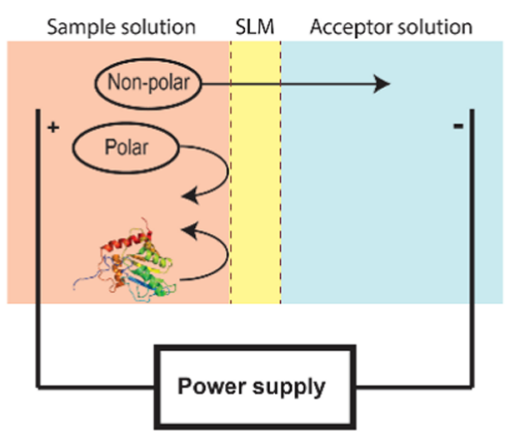

C

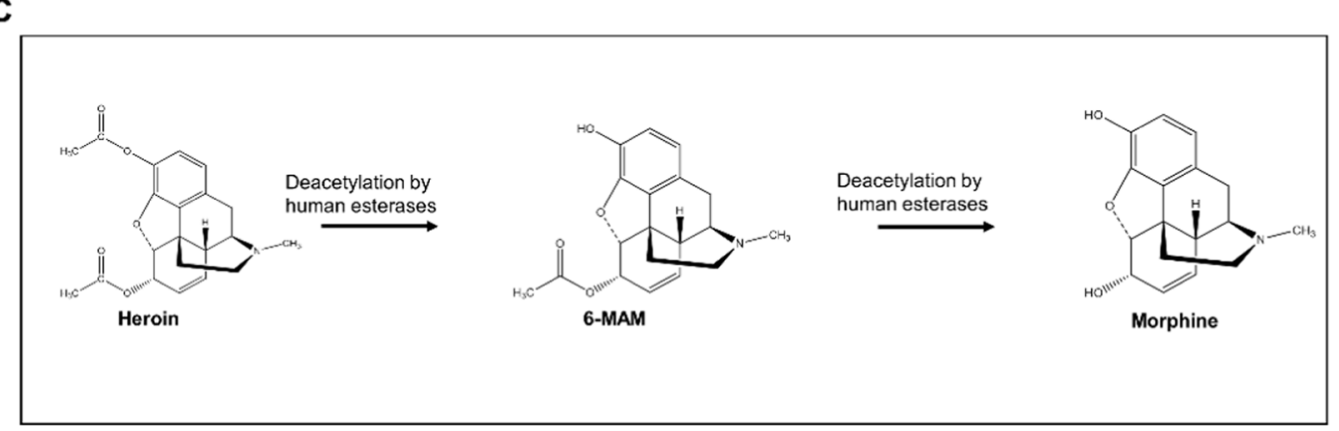

Figure 1. (A) Light microscopy picture of iPSC-derived liver organoids used in this study, scale bar $500 \mu \mathrm{m}$. (B) Electromembrane extraction (EME) principle. Charged analytes migrate from the donor solution (i.e., the sample solution) across the supported liquid membrane (SLM) and into the acceptor solution. Extraction selectivity is obtained by voltage polarity and partitioning into and through the SLM. Polar molecules and macromolecules are effectively discriminated from extraction by the hydrophobic SLM. (C) Illustration of well-documented liver phase I metabolism of heroin undergoing deacetylation to 6-MAM and morphine by human esterases (e.g., human liver carboxylesterase 1 and 2, hCE1 and hCE2, respectively).

metabolites with a high degree of selectivity. Prior to MS measurements, the compounds in the sample are separated by the LC system, allowing for increased sensitivity and selectivity.

There are few studies utilizing LC-MS for drug metabolism measurements of organoids. ${ }^{19-21}$ To the best of our knowledge, there are currently no studies dedicated to demonstrating the potential of drug metabolism studies with liver organoids and LC-MS. ${ }^{22}$ The key focus of this study is to show the potential of using liver organoids and LC-MS measurements as a methodology for drug metabolism studies. To ensure an efficient combination of organoids, LC-MS, and drug metabolism, several challenges must be addressed. The amounts of organoids can (depending on the production method) be quite limited per sample, requiring efficient sample preparation prior to analysis. It is also highly desirable that drug metabolism studies with organoids can be upscaled, which is difficult to combine with more standard sample preparation approaches which include centrifugation steps and manual pipetting (Figure S1A). In addition, liver organoids are grown in a complex medium (e.g., can contain $10 \%$ fetal bovine serum) requiring a thorough sample clean-up prior to LC-MS analysis. For extracting drugs, and the metabolites produced by organoids, we applied electromembrane extraction (EME; Figures 1B and S1B). In EME, an oil immobilized in the pores of a porous membrane (supported liquid membrane, SLM) is used to extract analytes from a cell medium (donor solution) to a protein-free MS-compatible acceptor solution. For the process, both aqueous compartments are $\mathrm{pH}$-adjusted to facilitate analyte ionization, and voltage is applied across the SLM. EME is therefore essentially an electrophoretic migration of ionized analytes across an oil membrane. ${ }^{23,24}$ Extraction selectivity is determined by both the partitioning of analytes into the SLM and the polarity and magnitude of the applied voltage. High clean-up efficiency of the target analytes can thus be achieved, and EME is highly successful in separating small-molecule drug substances from biological matrix substances, including salts, lipids, phospholipids, proteins, and blood cells. ${ }^{24,25}$ Such a clean-up is highly important prior to liquid chromatography-mass spectrometry to avoid ion suppression or enhancement. EME has recently advanced to the 96-well plate format ${ }^{26-28}$ (parallel-EME), and chip systems. ${ }^{29,30}$ Considering its documented traits regarding simple sample clean-up, we focus on using EME for organoids, which can be costly and limited in availability.

As a model system to show the potential of the methodology, we study the phase I metabolism of heroin to 6-monoacetylmorphine (6-MAM) and morphine (Figure 1C), as heroin liver metabolism is highly established, both with regards to the metabolizing enzymes ${ }^{31-33}$ (e.g., human liver carboxylesterase 1 and 2, hCE1 and hCE2, respectively), and the resulting metabolites. With the presented experiments, we have shown the proof of concept that liver organoids are EME compatible, and evaluate the advantages and challenges of parallel-EME/organoid/MS-based analysis for drug metabolism.

\section{EXPERIMENTAL SECTION}

Chemicals and Solutions. 2-Nitrophenyl octyl ether (NPOE), 2-nitrophenyl pentyl ether (NPPE), bis (2-ethylhexyl) hydrogen phosphite (DEHPi), bis(2-ethylhexyl) phosphate (DEHP), sodium hydroxide, ammonium formate (>99\%), formic acid (FA, reagent grade 95\%), L-ascorbic acid2 phosphate (AAP) were purchased from Sigma Aldrich (St. 

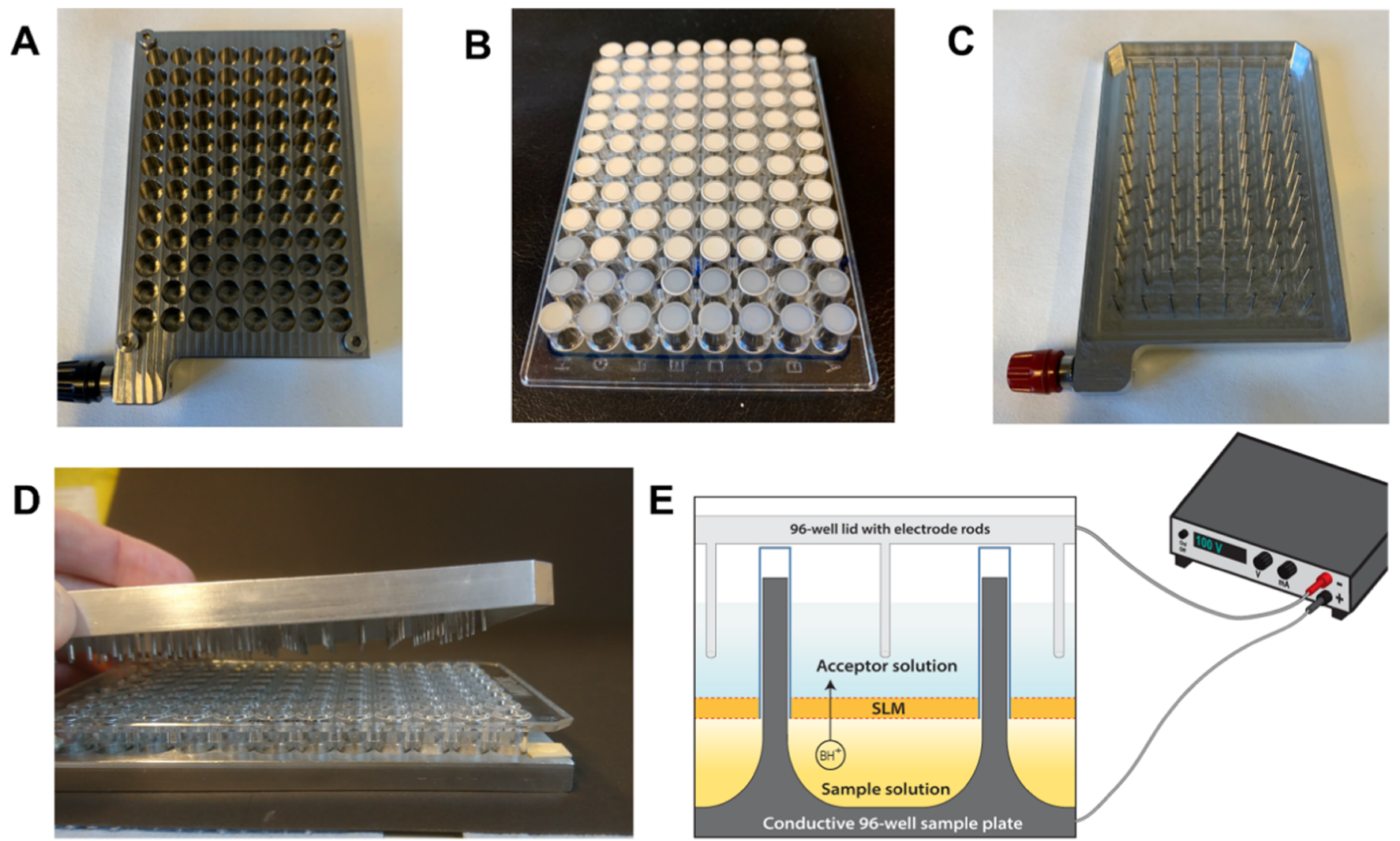

Figure 2. Experimental setup of 96-well parallel-EME. (A) Ninty-six well sample reservoir plate constituting the donor solution. (B) Ninty-six well filter plate, constituting the acceptor solution. (C) Aluminum lid with 96 electrode rods. (D) All plates clamped together. (E) Illustration of the extraction setup of parallel-EME coupled to the external power supply.

Louis, MO). LC-MS grade water and acetonitrile (ACN) were purchased from VWR (Radnor, PA). Chromasolv methanol (LC-MS grade) was from Honeywell Riedel-de Haën (Seelze, Germany). Heroin $\mathrm{HCl}, 6-\mathrm{MAM} \mathrm{HCl}$, and morphine were purchased from Lipomed AG (Arlesheim, Switzerland). Heroin- $d_{9}$, 6-MAM- $d_{6}$, and morphine- $d_{3}$ were purchased from Cerilliant (Austin, TX). Unless otherwise stated, the water used was type 1 water purified by a Milli-Q water purification system from Merck Millipore (Billerica, MA).

The 5 and $10 \mathrm{mM}$ ammonium formate buffer (w/v) was made by dissolving ammonium formate in LC-MS grade water followed by $\mathrm{pH}$ adjustment by the addition of $\mathrm{FA}$ to $\mathrm{pH}$ 3.1. A freshly made stock solution of $1 \mathrm{mM}$ heroin $\mathrm{HCl}$ in $0.9 \% \mathrm{NaCl}$ was made prior to each organoid experiment (stored at $4{ }^{\circ} \mathrm{C}$ ) and also used to prepare heroin calibration solutions. A stock solution of 6-MAM and morphine was prepared in $5 \mathrm{mM}$ ammonium formate buffer $\mathrm{pH} 3.1$ at a concentration of $50 \mu \mathrm{M}$ each and stored at $4{ }^{\circ} \mathrm{C}$. Two stock solutions of the internal standards heroin- $d_{9}$, 6-MAM- $d_{6}$, and morphine- $d_{3}$ were prepared in $5 \mathrm{mM}$ ammonium formate buffer $\mathrm{pH} 3.1$ with analyte concentration of $1.5 \mu \mathrm{M}$ each and $3 \mu \mathrm{M}$ each, respectively, and stored at $4{ }^{\circ} \mathrm{C}$.

Liver Organoid Differentiation from Induced Pluripotent Stem Cells. The iPSC cell line HPSI0114i-vabj_3 (Wellcome Trust Sanger Institute, Cambridgeshire, U.K.) was differentiated toward liver organoids using media from protocol by Ang et al. ${ }^{34}$ Briefly, the HPSI0114i-vabj_3 iPSC line was differentiated toward definitive endoderm in Iscove's modified Dulbecco's medium/F12 medium (Thermo Fisher Scientific, Waltham, MA) containing $3 \mu \mathrm{M}$ CHIR99021 (STEMCELL Technologies, Vancouver, Canada), 50 nM PI-
103 from Bio-Techne Ltd. (Abingdon, United Kingdom) and $100 \mathrm{ng} / \mathrm{mL}$ activin A (PeproTech, Cranbury, NJ) for one day and $100 \mathrm{ng} / \mathrm{mL}$ activin A for 2 more days. The definitive endoderm cells were subsequently treated with $1 \mu \mathrm{M}$ A8301 (Bio-Techne Ltd.), 10 ng/mL FGF2 (PeproTech), 30 ng/mL BMP4 (PeproTech), and $2 \mu \mathrm{M}$ all-trans retinoic acid (Sigma Aldrich) for one day, then with $10 \mathrm{ng} / \mathrm{mL}$ FGF2, $30 \mathrm{ng} / \mathrm{mL}$ BMP4, $1 \mu \mathrm{M}$ forskolin (PeproTech), $1 \mu \mathrm{M}$ Wnt-C59 (BioTechne Ltd.) for 2 more days and with $10 \mathrm{ng} / \mathrm{mL}$ FGF2, 30 $\mathrm{ng} / \mathrm{mL} \mathrm{BMP} 4,1 \mu \mathrm{M}$ forskolin for another day. On day 8 , the cells were detached and aggregated in the $\mathrm{U}$ bottom microwells in the presence of $20 \mathrm{ng} / \mathrm{mL}$ HGF (PeproTech), $10 \mathrm{ng} / \mathrm{mL}$ oncostatin M (OSM, PeproTech), 0.1 $\mu \mathrm{M}$ dexamethasone (Bio-Techne Ltd.), $1 \mu \mathrm{M}$ forskolin, $10 \mu \mathrm{g} / \mathrm{mL}$ human recombinant insulin (Sigma Aldrich), and $100 \mu \mathrm{M}$ AAP. After the formation of organoids at day 10, they were transferred into low attachment plates and cultured for another 10 days as free-floating organoids in William's $\mathrm{E}$ media (Thermo Fisher Scientific), supplemented with $10 \mathrm{ng} / \mathrm{mL}$ $\mathrm{HGF}$ and $10 \mathrm{ng} / \mathrm{mL}$ OSM, $10 \mu \mathrm{g} / \mathrm{mL}$ insulin, $100 \mu \mathrm{M}$ AAP, $0.1 \mu \mathrm{M}$ dexamethasone, $1 \mu \mathrm{M}$ forskolin, and $10 \mu \mathrm{M}$ DAPT (Bio-Techne Ltd.). The iPSC line AG2 $7^{35-38}$ was differentiated using a small-molecule-driven protocol that aims to sequentially mimic in vivo liver development, resulting in hepatocyte-containing liver organoids as described by Harrison et al. ${ }^{39}$

Liver Organoid Heroin Incubation. Prior to heroin incubation with organoids, $1 \mathrm{mM}$ heroin was diluted in the respective cell medium and sterilized by filtration using a 0.22 $\mu \mathrm{m}$ Millex-GV syringe filter (Merck Millipore). After 20 days of differentiation, from 20 to 60 organoids per well were 
treated with 10 or $50 \mu \mathrm{M}$ heroin in cell medium for $1,3,6$, and 24 h, respectively $(n=3)$, in separate Nunc flat-bottom 96-well microplates (Thermo Fisher Scientific). Metabolism was stopped by adding FA to a final concentration of $0.11 \mathrm{M}$, and the plates were frozen at $-80{ }^{\circ} \mathrm{C}$. In parallel, cell medium free from organoids $(n=3)$ were used as drug degradation control samples.

Parallel Electromembrane Extraction Setup. Prior to the extraction, $50 \mu \mathrm{L}$ of the heroin-exposed liver organoid samples (containing $0.11 \mathrm{M} \mathrm{FA}$ ) was added to $40 \mu \mathrm{L}$ of water and $10 \mu \mathrm{L}$ of the $1.5 \mu \mathrm{M}$ or $3 \mu \mathrm{M}$ internal standard solution. The samples were then loaded into the wells of an in-house built 96-well stainless steel plate (Figure 2A), previously described by Restan et al. ${ }^{28} \mathrm{~A}$ volume of $3 \mu \mathrm{L}$ of DEHP/ NPOE $(10 / 90, \mathrm{w} / \mathrm{w})$ was immobilized into the membrane pores $(0.45 \mu \mathrm{m}$ pore size $)$ of a 96-well MultiScreen-IP poly(vinylidene fluoride) (PVDF) filter plate from Merck Millipore (Figure 2B). The steel and filter plates were subsequently clamped together and $100 \mu \mathrm{L}$ of $10 \mathrm{mM}$ ammonium formate $\mathrm{pH} 3.1$ was loaded into each well of the filter plate, and thus constituting the acceptor solution. The filter plate was used to house the acceptor solution because the geometry of the steel plate wells provided better convection of the donor solution in this configuration, which improved the extraction kinetics. A conductive in-house built aluminum lid with 96 electrode rods (Figure 2C) was placed onto the filter plate, and the whole construct (Figure 2D) was placed on a Vibramax 100 Heidolph shaking board (Kellheim, Germany). The steel plate holding the organoid solution was connected to the anode of an external power supply (model ES 0300-0.45, Delta Elektronika BV, Zierikzee, The Netherlands), while the aluminum electrode lid was connected to the cathode (Figure 2E). Simultaneous extraction of all samples was performed for $15 \mathrm{~min}$ at $900 \mathrm{rpm}$ agitation, with $30 \mathrm{~V}$ applied for the first 2 min and $50 \mathrm{~V}$ applied for the remaining extraction duration. The stepped voltage was used to ensure that the extraction current was kept below $50 \mu \mathrm{A}$ per well, which was considered a safe limit for robust operation. ${ }^{40}$

Ultrahigh-Performance Liquid ChromatographyMass Spectrometry (UHPLC-MS). Determination of heroin, 6-MAM, and morphine was performed using UHPLC-MS based on a previously described method. ${ }^{41}$ The sample extracts were diluted $\times 10$ with $5 \mathrm{mM}$ ammonium formate $\mathrm{pH} 3.1$ and analyzed using an Acquity UHPLC pump coupled to a Xevo TQ (triple quadrupole) MS with electrospray ionization (ESI) interface, all from Waters (Milford, MA). The separation was achieved using the Acquity UPLC HSS T3 C18 column (2.1 $\mathrm{mm} \times 100 \mathrm{~mm}, 1.8 \mu \mathrm{m}$ particles). Solvent A consisted of 10 $\mathrm{mM}$ ammonium formate buffer $\mathrm{pH} 3.1$ and solvent $\mathrm{B}$ consisted of methanol. The sample injection volume was set to $7.5 \mu \mathrm{L}$, and the gradient elution was carried out at a flow rate of 0.5 $\mathrm{mL} / \mathrm{min}$ at $65{ }^{\circ} \mathrm{C}$ using the following gradient profile: from $0-$ $0.5 \mathrm{~min}$; $100 \%$ solvent A, $0.5-2.7 \mathrm{~min}$; 0-10\% solvent B, 2.7$3.3 \mathrm{~min} ; 10-20 \%$ solvent $B, 3.3-4.6 \mathrm{~min} ; 20-80 \%$ solvent $B$, 4.6-4.61 min; $80-100 \%$ solvent $\mathrm{B}, 4.61-6.60 \mathrm{~min} ; 100 \%$ solvent $\mathrm{B}, 6.60-6.61 \mathrm{~min} ; 100-0 \%$ solvent $\mathrm{B}, 6.61-7.50 \mathrm{~min}$; and $100 \%$ solvent $\mathrm{A}$. The capillary voltage was $3 \mathrm{kV}$, source temperature was $150{ }^{\circ} \mathrm{C}$, desolvation temperature was $500{ }^{\circ} \mathrm{C}$, and cone gas flow was $990 \mathrm{~L} / \mathrm{h}$. Detection was performed in positive mode using multiple reaction monitoring (MRM) with MS/MS transitions (MS/MS transition 1 being the quantifier and MS/MS transition 2 the qualifier) and collision energies for heroin $(\mathrm{m} / z 370>268$ at $30 \mathrm{eV}$ and $\mathrm{m} / z 370>$
211 at $38 \mathrm{eV}), 6-\mathrm{MAM}(m / z 328>165$ at $42 \mathrm{eV}$ and $m / z 328$ $>211$ at $30 \mathrm{eV})$, morphine $(\mathrm{m} / z 286>201$ at $24 \mathrm{eV}$ and $\mathrm{m} / z$ $286>165$ at $42 \mathrm{eV})$, heroin- $d_{9}(m / z 379>272$ at $30 \mathrm{eV}), 6-$ MAM- $d_{6}(m / z 334>165$ at $42 \mathrm{eV})$, and morphine- $d_{3}(289>$ 165 at $30 \mathrm{eV})$. Data was acquired and processed using MassLynx 4.1 software (Waters).

Nanoliquid Chromatography-Mass Spectrometry (nanoLC-MS). The nanoLC-MS setup consisted of a TSQ Quantiva, triple quadrupole MS, the nanoFlex ESI ion source, and the EASY-nLC 1000 or 1200 pump equipped with an autosampler, all from Thermo Fisher. Acclaim PepMap 100 C18 (3 $\mu \mathrm{m}$ particle size $)$ pre- $(75 \mu \mathrm{m}$ inner diameter, ID, and $20 \mathrm{~mm}$ length $)$ and analytical $(75 \mu \mathrm{m}$ ID $\times 50 \mathrm{~mm})$ columns from Thermo Fisher Scientific were used for the chromatographic separation. In-house made ${ }^{42}$ analytical columns were packed with $3 \mu \mathrm{m}$ Atlantis T3 particles (Waters) or $2.6 \mu \mathrm{m}$ Accucore particles (Thermo Fisher Scientific) in fused silica capillaries of $75 \mu \mathrm{m}$ ID from Polymicro Technologies (Phoenix, AZ). The analytical column was coupled to a 40 $\mathrm{mm}$ stainless steel emitter (20 $\mu \mathrm{m}$ ID) purchased from Thermo Fisher. The extracted organoid samples (AG27 iPSC derived) were further diluted $\times 10^{3}$ in $5 \mathrm{mM}$ of ammonium formate $\mathrm{pH} 3.1$ buffer, and the injection volume was set to 2 $\mu \mathrm{L}$. The nanoLC pump was equipped with two solvent compartments (A and B), where A contained $0.1 \% \mathrm{FA}$ in the LC-MS grade water $(\mathrm{v} / \mathrm{v})$ and $\mathrm{B}$ contained $0.1 \% \mathrm{FA}$ in the LCMS grade water and ACN $(10 / 90, v / v)$. The gradient elution was carried out with $3-50 \% \mathrm{~B}$ in 8 min with a constant flow rate of $500 \mathrm{~nL} / \mathrm{min}$. The spray voltage was set to $2.2 \mathrm{kV}$ and the ion transfer tube temperature was set to $310^{\circ} \mathrm{C}$. Detection was performed in positive mode using MRM with MS/MS transitions and collision energies for heroin $(\mathrm{m} / z 370>268$ at $38 \mathrm{eV}$ and $370>211$ at $41 \mathrm{eV}), 6-\mathrm{MAM}(\mathrm{m} / z 328>165$ at 48 $\mathrm{eV}$ and $328>211$ at $36 \mathrm{eV})$, morphine $(\mathrm{m} / z 286>181$ at 48 $\mathrm{eV}$ and $286>165$ at $51 \mathrm{eV})$, heroin- $d_{9}(\mathrm{~m} / z 379>272$ at 38 $\mathrm{eV}$ and $379>211$ at $41 \mathrm{eV}), 6-\mathrm{MAM}-d_{6}(m / z 334>211$ at 35 $\mathrm{eV}$ and $334>165$ at $48 \mathrm{eV})$, and morphine- $d_{3}(\mathrm{~m} / z 289>181$ at $48 \mathrm{eV}$ and $289>165$ at $51 \mathrm{eV})$.

For a one-column setup, the pump outlet was coupled to an external six-port valve from Valco Instruments Company Inc (VICI, Houston, TX) equipped with a $75 \mu \mathrm{m}$ ID $\times 11 \mathrm{~cm}$ fused silica injection loop $(500 \mathrm{~nL})$, a nut with a syringe sleeve and a $75 \mu \mathrm{m} \mathrm{ID} \times 10 \mathrm{~cm}$ fused silica capillary waste outlet. The flow outlet from the six-port valve was coupled to a stainless steel tee-piece (VICI) through a $20 \mu \mathrm{m} \times 40 \mathrm{~cm}$ fused silica capillary from Polymicro Technologies using stainless steel nuts and vespel/graphite ferrules (VICI). The analytical column inlet was coupled to the stainless steel tee-piece, also coupled to a plug through a $550 \mathrm{~mm}$ nanoViper $(75 \mu \mathrm{m} \mathrm{ID}$, Thermo Fisher). A $500 \mu \mathrm{L}$ syringe $(51 \mathrm{~mm})$ from Hamilton (Reno, Nevada) was used to load the samples. Xcalibur version 2.2 was used to obtain chromatograms and mass spectra (Thermo Fisher).

Protein Profiling by Nanoliquid ChromatographyMass Spectrometry. Acetone precipitated AG27 iPSCderived liver organoid protein samples were subjected to sodium dodecyl sulfate-polyacrylamide gel electrophoresis (SDS-PAGE) gel electrophoresis, and the gel lanes were sliced into five sample fractions and digested with trypsin as previously described. ${ }^{43}$ The peptide solutions were desalted using OMIX C18-micro solid-phase extraction (SPE) columns (Agilent, Santa Clara, CA). A Q-Exactive mass spectrometer (Thermo Fisher Scientific) equipped with a nanoFlex nano- 
spray ion source was used for the nanoLC-MS analyses, coupled to an EASY-nLC 1000 pump (Thermo Fisher). Peptide separation was achieved using Acclaim PepMap 100 pre- $(20 \mathrm{~mm})$ and separation columns $(250 \mathrm{~mm})$ of $75 \mu \mathrm{m}$ inner diameter and $3 \mu \mathrm{m}$ particles (Thermo Fisher). Solvent A was $0.1 \%$ FA in LC-MS grade water $(\mathrm{v} / \mathrm{v})$ and solvent B was $0.1 \%$ FA in LC-MS grade water and ACN (5/95, v/v). Peptides were separated using a $180 \mathrm{~min}$ long gradient ranging from $3-15 \%$ solvent B (after optimization with the predigested HeLa samples from Thermo Fisher). The mass spectrometer was run in a positive mode with full MS $(\mathrm{m} / z=400-2000)$ and data-dependent tandem mass spectrometry (ddMS2) with top $\mathrm{N}$ set to be 10 ions. Raw files were processed and database searches performed with Proteome Discoverer 2.3 (Thermo Fisher Scientific), using MASCOT version 2.4 to search the SwissProt database (human, 20431 entries). Proteins were identified with the following settings; peptide identification with a false discovery rate (FDR) threshold of $\leq 0.01$, protein identification with a FDR threshold of $\leq 0.01$ (strict) and $\leq 0.05$ (relaxed) and digestion by trypsin with at most one missed cleavage. Dynamic modification was set to be oxidation and acetyl (N-term), static modification was set to be carbamidomethyl. Information on the elution profile and fragment match spectrum of each of the identified peptides for hCES1 (accession number P23141), hCES2 (also called cocaine esterase, accession number O00748), and UDPglucuronosyltransferase 2B7 (accession number P16662) were obtained and verified by comparison with the raw file.

Calculation of Recovery. Recovery measurements were performed using capillary electrophoresis with ultraviolet spectroscopy detection (CE-UV) (see Supporting Information for experimental description) with an initial analyte concentration of $5 \mu \mathrm{M}$. The recovery (\%) was calculated using the following formula

$$
R(\%)=\frac{A_{\text {final }}}{A_{\text {initial }}} \times 100 \%
$$

where $A_{\text {final }}$ and $A_{\text {initial }}$ are the area of analyte collected in the acceptor solution and the area of the analyte originally present in the sample.

\section{RESULTS AND DISCUSSION}

In this study, several analytical approaches were evaluated for liver organoid drug measurements. With the future objective of advancing to online analyses, EME was assessed in a 96-well format (parallel-EME) for the high-throughput clean-up of analytes from the organoid cell medium, a method previously shown to enable selective and fast extraction from complex matrices (and also on-chip). ${ }^{44}$ A conventional UHPLC-MS method used for clinical routine analyses was applied to explore the heroin-metabolizing properties of the parallel-EME extracted liver organoids. To get an understanding of the heroin-metabolizing liver enzymes present in the organoids, an untargeted proteomic case study using nanoLC-MS was undertaken. Lastly, two analytical approaches more suitable for online action, limited samples, and increased sensitivity were evaluated: CE, which is widely established for rapid onchip separations, ${ }^{45-47}$ and nanoLC-MS, which allows high sensitivity measurements. ${ }^{48}$

Parallel Electromembrane Extraction Optimization for Heroin and Metabolites. To evaluate the potential of MS for the analysis of liver organoids, heroin was chosen as a model substance due to its familiar phase I metabolism to 6MAM and morphine in the liver. To our knowledge, heroin metabolism of organoids has not previously been studied with mass spectrometric-based techniques. Although morphine extraction with EME has previously been performed, ${ }^{49-51}$ the extraction of heroin and 6-MAM with EME has, to our knowledge, not previously been performed. Therefore, parallelEME conditions focusing on these three compounds were initially assessed. The experimental conditions (Figure 3) were
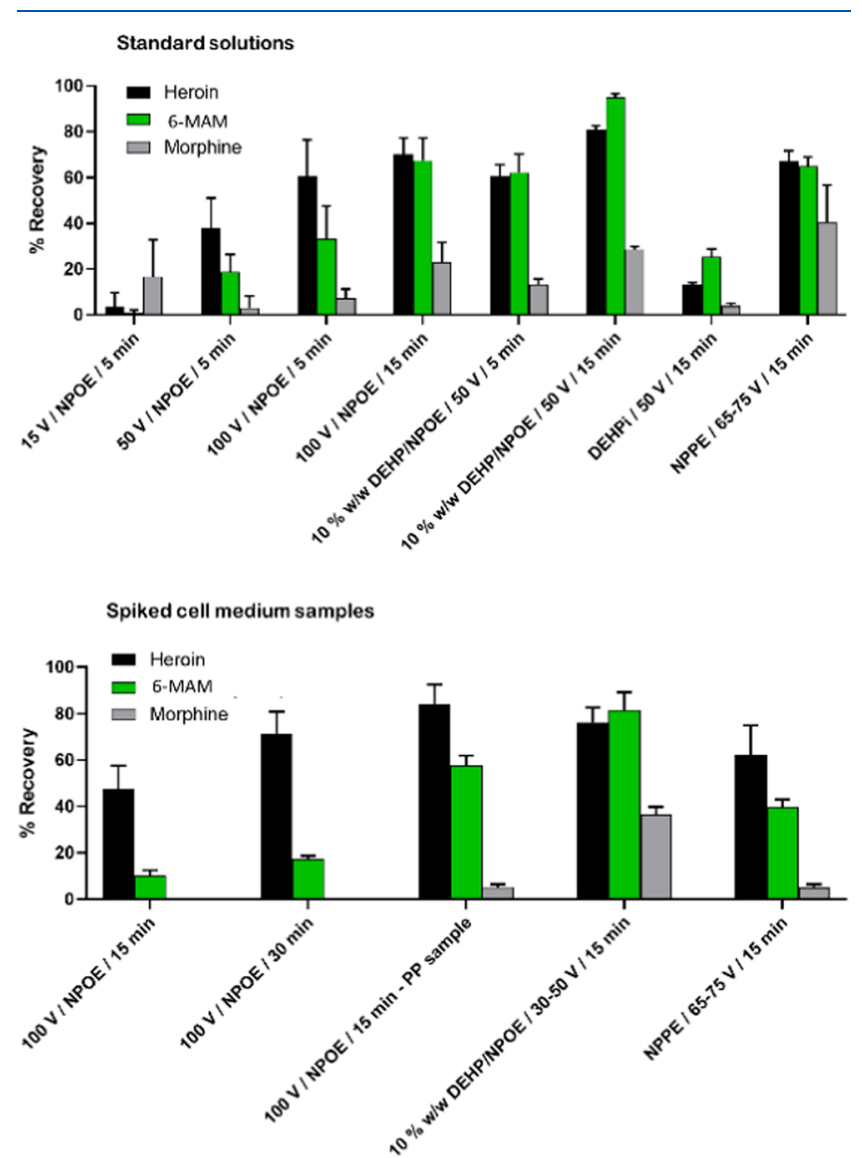

Figure 3. Analyte recovery (\%) of parallel-EME under varying conditions (SLM composition, extraction voltage, and extraction time), with $5 \mu \mathrm{M}$ standard solutions and spiked cell medium samples using CE-UV for quantitation.

selected based on previous experience and literature reports. ${ }^{49,52,53}$ Due to the difference in the polarity of the analytes, $>30 \%$ recovery and $<15 \%$ RSD were set as the acceptance criteria of extraction performance. Best recovery and repeatability for analytes in both standard solutions and spiked cell medium samples were obtained using a parallelEME system comprising 10\% (w/w) DEHP/NPOE as SLM, an extraction time of $15 \mathrm{~min}$, and an extraction voltage of $50 \mathrm{~V}$. From the cell medium, these conditions gave recoveries of $76 \%$ (heroin), 82\% (6-MAM), and 36\% (morphine) and RSD $<10 \%$, which was considered acceptable for the current application. With these parameters, the average extraction current was $<50 \mu \mathrm{A}$ per well throughout the extraction. The extraction method was therefore not optimized any further. Elevated voltages could possibly improve the recoveries but can potentially also result in analyte degradation. In addition, for increasing the accuracy, correction for nonexhaustive 


\section{Heroin}

A
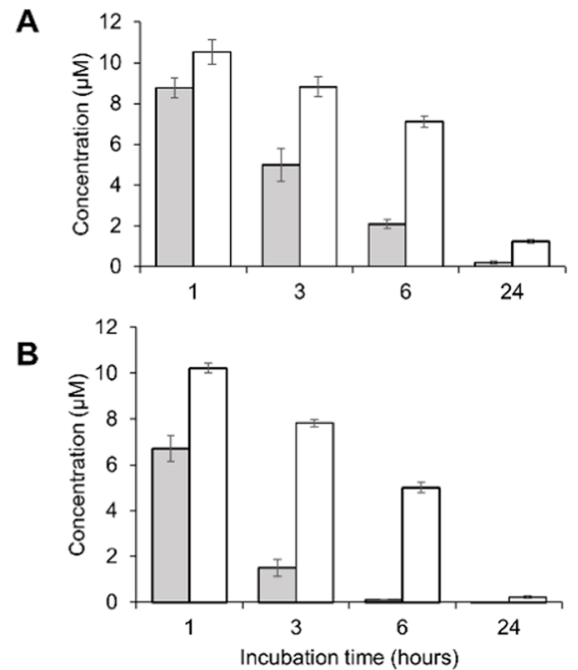

6-MAM
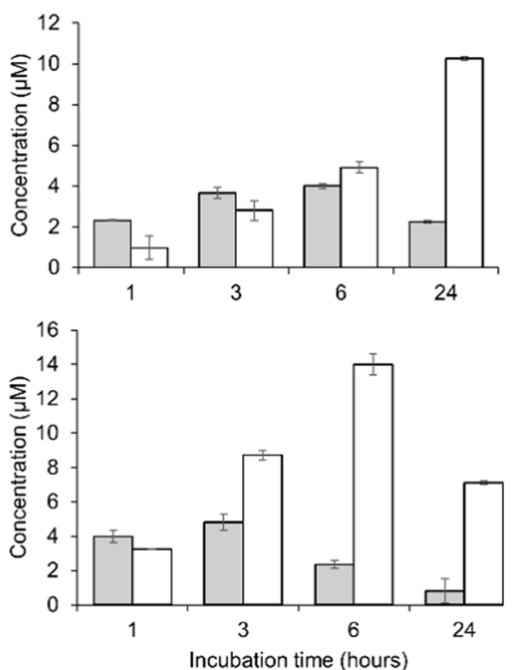

Morphine
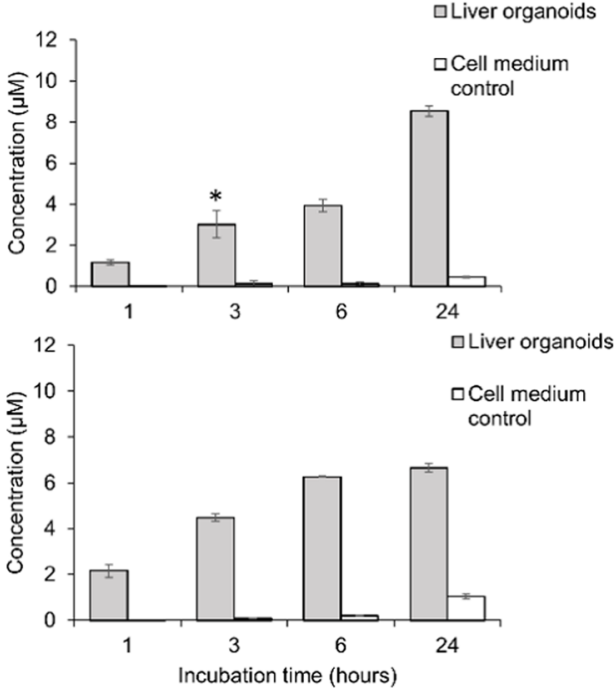

Figure 4. Concentration of heroin and metabolites in a study of liver organoid drug metabolism using parallel-EME and UHPLC-MS after incubation of liver organoids differentiated from the iPSC cell lines (A) AG27 (60 organoids) and (B) HPSI0114i-vabj_3 (20 organoids) in $10 \mu \mathrm{M}$ heroin for 1, 3, 6, and $24 \mathrm{~h}$. In parallel, cell medium free from organoids was used as the drug degradation control sample. Each bar represents the mean $( \pm S D)$ of triplicate samples. One of the three replicates of time point $6 \mathrm{~h}$ liver organoids (HPSI0114i-vabj_3) was discarded. The asterisk indicates the removal of one data point due to a poor internal standard signal.

extractions was done by spiking the samples with isotopically labeled internal standards prior to extraction.

Parallel Electromembrane Extraction of Liver Organoid Heroin Metabolites. Samples containing 20 and 60 liver organoids per well were exposed to $10 \mu \mathrm{M}$ heroin for 1,3 , 6 , and $24 \mathrm{~h}$. With the exception of 6-MAM and heroin at time point $24 \mathrm{~h}$, the sample-to-sample repeatability was $0.4-25 \%$ with the two organoid iPSC sources (Figure 4A,B). Heroin levels decreased with time to 6-MAM (both enzymatic and nonenzymatic), and with subsequent enzymatic metabolism to morphine, adding to the confirmation that the liver organoids had traits related to human livers. Similar heroin metabolism kinetics was also observed for liver organoids derived from hepatocytes from one patient case (see Figure S2). However, the kinetics were (expectedly) substantially slower than that observed with, e.g., high enzyme-availability microsomes and S-9 fraction, ${ }^{17,54}$ see Figure S3; although parallel-EME and MS are compatible with phase I metabolism monitoring, we were not able to observe phase II metabolites morphine-3glucuronide (M3G) and morphine-6-glucuronide (M6G). Traces of these metabolites could however be observed when employing more manual, centrifugation-based sample preparations (Figure S4). A key reason is a weakness of EME, that highly polar compounds have low recovery; this can in many cases be fine-tuned. ${ }^{53,55}$

To complement the observations of the liver organoids enzymatic heroin-metabolizing properties, a case study using MS-based untargeted proteomics was undertaken. We could identify the presence of proteotypic peptides (FDR $\leq 1 \%$ ) related to the key liver enzymes ${ }^{56-60}$ hCES1 (9 peptides identified) and hCES2 (4 peptides identified) in the organoids differentiated from the iPSC cell line AG27 (Figure 5A-C, see also Table S1 for peptide overview). Also, one peptide was identified related to one of the heroin phase II metabolism enzymes, ${ }^{33,57}$ UDP-glucuronosyltransferase 2B7 (Table S1).

Compatibility of Organoid EME Extracts with Various Separation Techniques. The organoid EME extracts were analyzed using UHPLC-MS instrumentation, which provided high-resolution separations within $5 \mathrm{~min}$ (Figure S5). We have also investigated other separation approaches that can be compatible with small samples and online action. Capillary electrophoresis, perhaps the most "chip-ready" of the techniques investigated, was capable of fast separations of organoid extracts (separation within $2.5 \mathrm{~min}$ ) and low sample consumption (injection volume equivalent to $107 \mathrm{~nL}$ ), with these initial experiments demonstrated with simple UV detection (Figure S6). However, organoid incubation in 50 $\mu \mathrm{M}$ heroin was needed to achieve detection with CE-UV, and thus no further quantification of the analytes could be performed.

The limit of quantification (LOQ) for UHPLC-MS measurements in this study was $1 \mathrm{nM}(7 \mu \mathrm{L}$ injection volume). NanoLC, a sensitive approach that has been mostly associated with proteomics in recent years, was seen to provide $0.95 \mathrm{pM}$ detection ( $1 \mu \mathrm{L}$ injection volume) for some smallmolecule analytes such as heroin (results not shown). For the more hydrophobic analytes heroin and 6-MAM, the organoid extracts analyzed with nanoLC-MS could thus be 1000 times more diluted compared to that of UHPLC-MS analysis without compromising the chromatographic performance or sensitivity (Figure 6A). However, poor performance was associated with the nanoLC-MS analysis of morphine, the most polar of the metabolites observed; the chromatographic peak was completely absent in the chromatograms of the organoid extracts (Figure 6A) and sporadically very deformed or absent in that of standard solutions. This was the case for large volume injection, both using on-column injection and an SPE column. We also examined the in-house-packed nano reversed-phase (RP) LC columns which were more compatible with highly aqueous mobile phases (Accucore and Atlantis T3), but poor peak shape and breakthrough/poor retention time repeatability were still issues. Various parameters were tested, e.g., sample loading time and maximum sample loading pressure (of the Thermo nano pumps). To illustrate these 

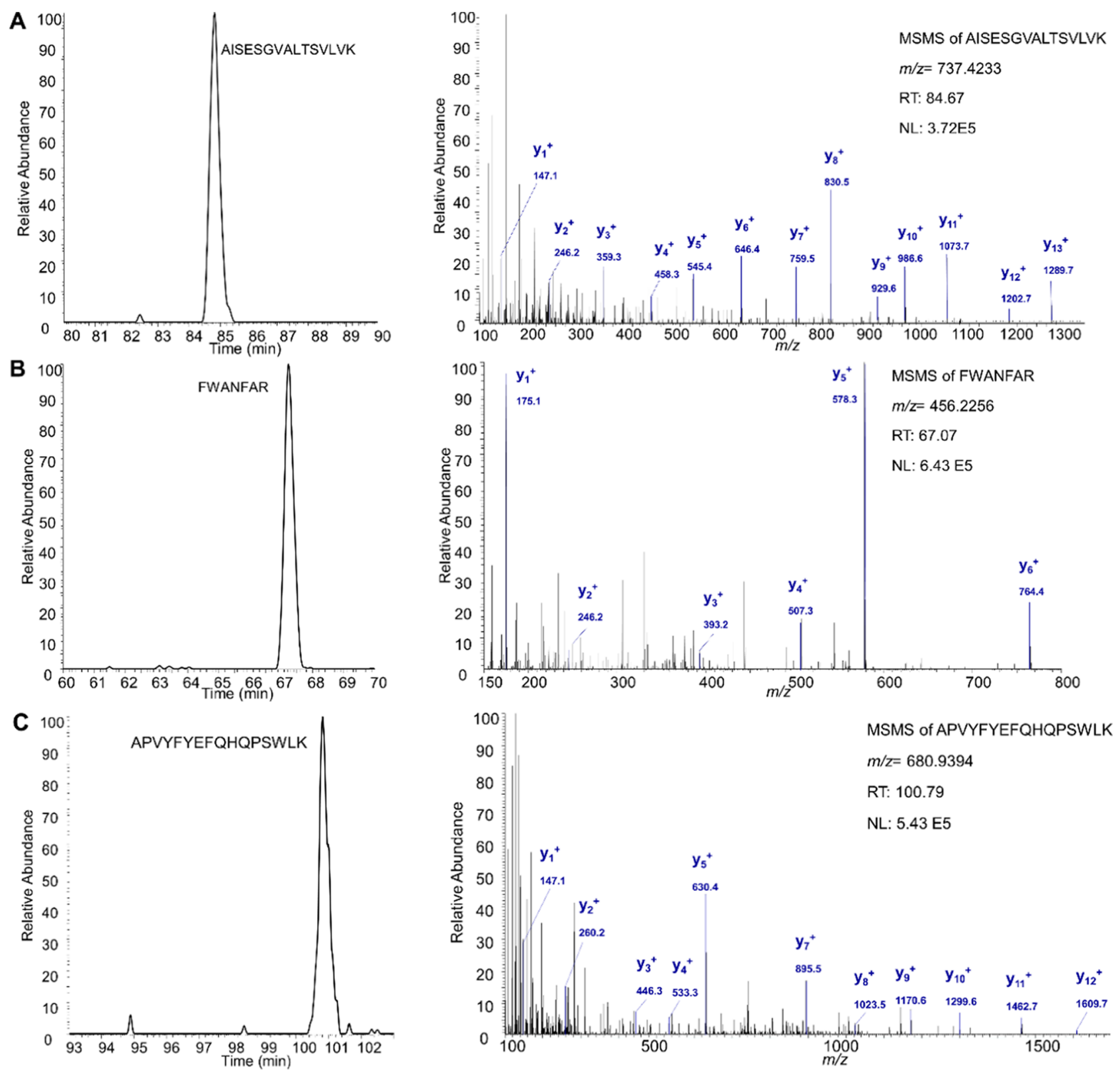

Figure 5. Total ion chromatogram of identified peptides (left) and the respective peptide fragmentation spectrum (right) of enzymes related to heroin liver phase I metabolism. (A) Peptide AISESGVALTSVLVK $(m / z$ 737.42) from hCES1, identified at charge +2 . (B) Peptide FWANFAR $(m / z$ 456.23) from hCES1, identified at charge +2 . (C) Peptide APVYFYEFQHQPSWLK $(m / z 680.94)$ from hCES2, identified at charge +3 .

effects, see Figure 6B, which shows that several loading times were suited for 6-MAM and heroin using on-column injection, but none were suited for morphine.

\section{CONCLUSIONS}

Liver organoids and LC-MS measurements are a promising concept for drug metabolism studies, here demonstrated for heroin phase I metabolism. This concept can be well suited for drug metabolism studies of other drugs, and direct measurements of drug metabolism could also provide valuable insight when optimizing organoid development protocols. A proteomic case study using nanoLC-MS identified proteotypic peptides from heroin-metabolizing enzymes, complementing the observations of the liver organoids enzymatic heroinmetabolizing properties. EME-MS was shown to be a promising combination for the liver organoid-based analysis of drug metabolism. EME in a 96-well format (parallel-EME) was used to extract heroin and metabolites from various organoids in a complex medium, followed by UHPLC-MS measurements. In addition, the chromatographic performance was not perturbed by the initial complex matrix (analyte retention time repeatability with a maximum RSD of $0.07 \%$ ), suggesting that parallel-EME was a suitable basis for organoidderived sample preparation. It is reasonable to assume that the approach can also be applicable to other organoid variants, e.g., kidney and heart. Parallel-EME was indeed an approach that allowed multiple samples to be simply handled, more so than standard approaches to related tissues (centrifugations, several sample pipetting steps), which can allow higher throughput in larger-scale studies. We are currently developing 96-well plates made of conductive polymers, which we believe will be suited for both cell studies and EME; this will reduce yet another step 
A

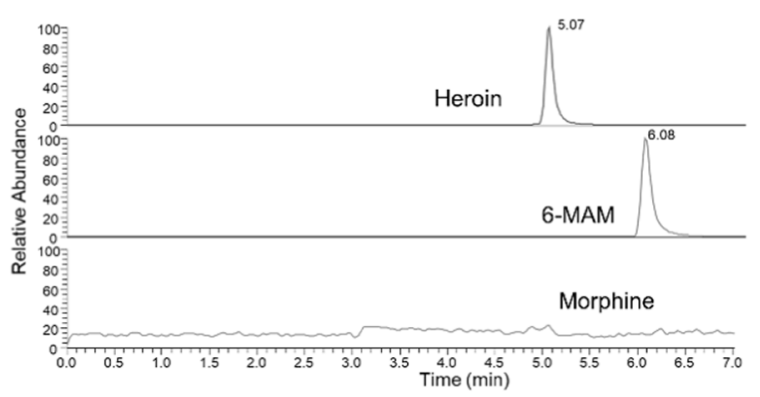

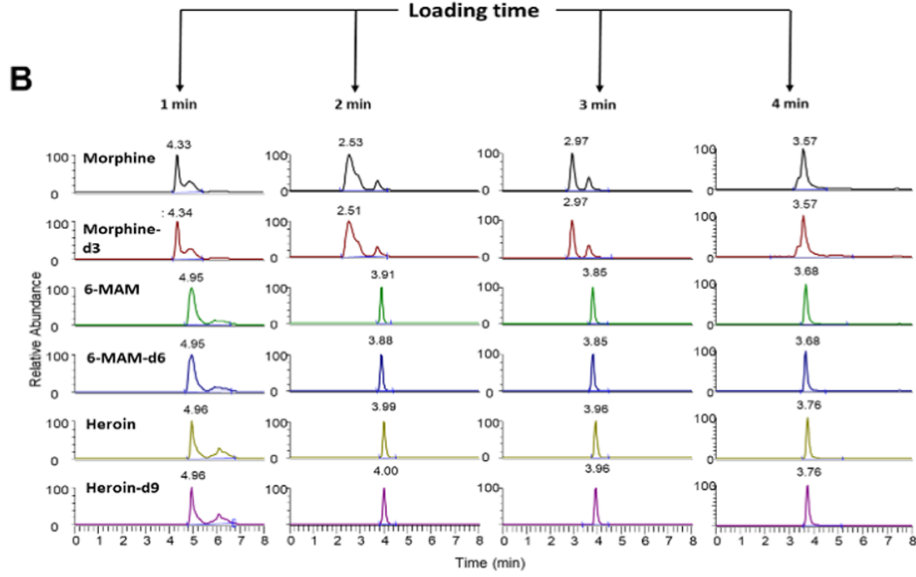

Figure 6. (A) MRM chromatograms of heroin, 6-MAM, and morphine in the extracted liver organoid sample treated with $10 \mu \mathrm{M}$ heroin for $1 \mathrm{~h}$ (AG27). The sample was analyzed using a two-column setup with Acclaim PepMap columns and injection volume of $2 \mu \mathrm{L}$. (B) MRM chromatograms of a $375 \mathrm{nM}$ standard solution containing heroin, morphine, 6-MAM, and their corresponding internal standards were analyzed using the one-column setup equipped with an Acclaim PepMap commercially packed analytical column with different on-column loading times ( 1 , 2,3 , and $4 \mathrm{~min}$ ), and injection volume of $500 \mathrm{~nL}$.

of sample handling. One disadvantage that needs to be addressed is the difficulty in extracting very polar metabolites with EME, and further optimizations will therefore continue.

Following this proof-of-concept study, we will continue to explore iterations of the here presented EME configuration with the aim of further increasing the sensitivity while retaining robustness and scalability; a natural next step will be nanoliterscale online EME-LC-MS of organoid-derived samples. Related systems have been demonstrated with microsomes, ${ }^{30}$ but those systems require larger separation columns and are arguably not suited for trace samples. Due to challenges with nanoLC, we will instead likely investigate the use of capillary LC or microbore LC as a compromise between sensitivity and robustness.

\section{ASSOCIATED CONTENT}

\section{(s) Supporting Information}

The Supporting Information is available free of charge at https://pubs.acs.org/doi/10.1021/acs.analchem.0c05082.

Schematic overview of the workflow of drug studies performed with the sample preparation approaches centrifugation and Pa-EME, liver organoid drug metabolism using Pa-EME and UHPLC-MS after incubation of patient-derived liver organoids in heroin, heroin metabolism in microsomes and S-9 fractions using UHPLC-MS, liver organoid drug phase I and II metabolism using centrifugation, and UHPLC-MS, a table on the peptides identified of the enzymes hCES1, hCES2, and UDP-glucuronosyltransferase 2B7, chromatogram showing the separation of the analytes M3G, M6G, morphine, 6-MAM, and heroin with the use of UHPLC-MS, electropherogram of liver organoid drug phase I metabolism using Pa-EME and CE-UV, and the following additional experimental procedures: heroin metabolism assay in S-9 fractions and microsomes, sample preparation using centrifugation, and CE-UV setup (PDF)

\section{AUTHOR INFORMATION}

\section{Corresponding Author}

Steven Ray Wilson - Department of Chemistry and Hybrid Technology Hub-Centre of Excellence, Institute of Basic Medical Sciences, Faculty of Medicine, University of Oslo, NO-0315 Oslo, Norway; ๑ orcid.org/0000-0002-97551188; Phone: +47 97010953; Email: stevenw@ kjemi.uio.no

\section{Authors}

Frøydis Sved Skottvoll - Department of Chemistry and Hybrid Technology Hub-Centre of Excellence, Institute of Basic Medical Sciences, Faculty of Medicine, University of Oslo, NO-0315 Oslo, Norway; @ orcid.org/0000-00025299-2502

Frederik André Hansen - Department of Pharmacy, University of Oslo, NO-0316 Oslo, Norway; ๑ orcid.org/ 0000-0002-1666-0447

Sean Harrison - Hybrid Technology Hub-Centre of Excellence, Institute of Basic Medical Sciences, Faculty of Medicine, University of Oslo, NO-0317 Oslo, Norway

Ida Sneis Boger - Department of Chemistry and Hybrid Technology Hub-Centre of Excellence, Institute of Basic Medical Sciences, Faculty of Medicine, University of Oslo, NO-0315 Oslo, Norway

Ago Mrsa - Department of Chemistry and Hybrid Technology $\mathrm{Hub}$-Centre of Excellence, Institute of Basic Medical Sciences, Faculty of Medicine, University of Oslo, NO-0315 Oslo, Norway

Magnus Saed Restan - Department of Pharmacy, University of Oslo, NO-0316 Oslo, Norway

Matthias Stein - Institute of Medicinal and Pharmaceutical Chemistry, TU Braunschweig, DE-38106 Braunschweig, Germany

Elsa Lundanes - Department of Chemistry, University of Oslo, NO-0315 Oslo, Norway

Stig Pedersen-Bjergaard - Department of Pharmacy, University of Oslo, NO-0316 Oslo, Norway; Department of Pharmacy, Faculty of Health and Medical Sciences, University of Copenhagen, 2100 Copenhagen, Denmark; (1) orcid.org/0000-0002-1666-8043 
Aleksandra Aizenshtadt - Hybrid Technology Hub-Centre of Excellence, Institute of Basic Medical Sciences, Faculty of Medicine, University of Oslo, NO-0317 Oslo, Norway

Stefan Krauss - Hybrid Technology Hub-Centre of Excellence, Institute of Basic Medical Sciences, Faculty of Medicine, University of Oslo, NO-0317 Oslo, Norway; Department of Immunology and Transfusion Medicine, Oslo University Hospital, 0317 Oslo, Norway

Gareth Sullivan - Hybrid Technology Hub-Centre of Excellence, Institute of Basic Medical Sciences, Faculty of Medicine, University of Oslo, NO-0317 Oslo, Norway; Department of Pediatric Research, Oslo University Hospital and University of Oslo, 0317 Oslo, Norway

Inger Lise Bogen - Section for Drug Abuse Research, Department of Forensic Sciences, Oslo University Hospital, NO-0424 Oslo, Norway; Institute of Basic Medical Sciences, Faculty of Medicine, University of Oslo, NO-0317 Oslo, Norway

Complete contact information is available at: https://pubs.acs.org/10.1021/acs.analchem.0c05082

\section{Notes}

The authors declare no competing financial interest.

\section{ACKNOWLEDGMENTS}

This work was supported by the Research Council of Norway through its Centre of Excellence Scheme, project number 262613. The work was also supported by the Olav Thon Foundation. Financial support from UiO:Life Science is also gratefully acknowledged. Technical assistance by Elisabeth Nerem (Department of Forensic Sciences, Oslo University Hospital, Oslo, Norway) was greatly appreciated. S.R.W. is a member of the National Network of Advanced Proteomics Infrastructure (NAPI), which is funded by the Research Council of Norway INFRASTRUKTUR-program (project number: 295910).

\section{REFERENCES}

(1) Prakash, C.; Shaffer, C. L.; Nedderman, A. Mass Spectrom. Rev. 2007, 26, 340-369.

(2) Au, S. H.; Chamberlain, M. D.; Mahesh, S.; Sefton, M. V.; Wheeler, A. R. Lab Chip 2014, 14, 3290-3299.

(3) Matthews, H.; Hanison, J.; Nirmalan, N. Proteomes 2016, 4, No. 28.

(4) Lancaster, M. A.; Knoblich, J. A. Science 2014, 345, No. 1247125.

(5) Clevers, H. Cell 2016, 165, 1586-1597.

(6) Rossi, G.; Manfrin, A.; Lutolf, M. P. Nat. Rev. Genet. 2018, 19, 671-687.

(7) Pampaloni, F.; Reynaud, E. G.; Stelzer, E. H. K. Nat. Rev. Mol. Cell Biol. 2007, 8, 839-845.

(8) Westmoreland, C.; Holmes, A. M. Organogenesis 2009, 5, 67-72. (9) Lancaster, M. A.; Renner, M.; Martin, C.-A.; Wenzel, D.; Bicknell, L. S.; Hurles, M. E.; Homfray, T.; Penninger, J. M.; Jackson, A. P.; Knoblich, J. A. Nature 2013, 501, 373-379.

(10) Stevens, K. R.; Kreutziger, K. L.; Dupras, S. K.; Korte, F. S.; Regnier, M.; Muskheli, V.; Nourse, M. B.; Bendixen, K.; Reinecke, H.; Murry, C. E. Proc. Natl. Acad. Sci. U.S.A. 2009, 106, 16568-16573.

(11) van de Wetering, M.; Francies; Hayley, E.; Francis; Joshua, M.; Bounova, G.; Iorio, F.; Pronk, A.; van Houdt, W.; van Gorp, J.; Taylor-Weiner, A.; Kester, L.; McLaren-Douglas, A.; Blokker, J.; Jaksani, S.; Bartfeld, S.; Volckman, R.; van Sluis, P.; Li; Vivian, S. W.; Seepo, S.; Sekhar Pedamallu, C.; Cibulskis, K.; et al. Cell 2015, 161, 933-945.

(12) Huch, M.; Dorrell, C.; Boj, S. F.; van Es, J. H.; Li, V. S. W.; van de Wetering, M.; Sato, T.; Hamer, K.; Sasaki, N.; Finegold, M. J.;
Haft, A.; Vries, R. G.; Grompe, M.; Clevers, H. Nature 2013, 494, 247-250.

(13) Park, E.; Kim, H. K.; Jee, J.; Hahn, S.; Jeong, S.; Yoo, J. Toxicol. Appl. Pharmacol. 2019, 385, No. 114790.

(14) Huch, M.; Gehart, H.; van Boxtel, R.; Hamer, K.; Blokzijl, F.; Verstegen; Monique, M. A.; Ellis, E.; van Wenum, M.; Fuchs; Sabine, A.; de Ligt, J.; van de Wetering, M.; Sasaki, N.; Boers; Susanne, J.; Kemperman, H.; de Jonge, J.; Ijzermans, J. N. M.; Nieuwenhuis, E. E. S.; Hoekstra, R.; Strom, S.; Vries, R. R. G.; et al. Cell 2015, 160, 299312.

(15) Driehuis, E.; Kretzschmar, K.; Clevers, H. Nat. Protoc. 2020, 15, 3380-3409.

(16) Jia, L.; Liu, X. Curr. Drug Metab. 2007, 8, 822-829.

(17) Brandon, E. F. A.; Raap, C. D.; Meijerman, I.; Beijnen, J. H.; Schellens, J. H. M. Toxicol. Appl. Pharmacol. 2003, 189, 233-246.

(18) Greek, R.; Menache, A. Int. J. Med. Sci. 2013, 10, 206-221.

(19) Yoshida, S.; Miwa, H.; Kawachi, T.; Kume, S.; Takahashi, K. Sci. Rep. 2020, 10, No. 5989.

(20) Mills, R. J.; Parker, B. L.; Quaife-Ryan, G. A.; Voges, H. K.; Needham, E. J.; Bornot, A.; Ding, M.; Andersson, H.; Polla, M.; Elliott, D. A.; Drowley, L.; Clausen, M.; Plowright, A. T.; Barrett, I. P.; Wang, Q.-D.; James, D. E.; Porrello, E. R.; Hudson, J. E. Cell Stem Cell 2019, 24, 895-907.

(21) Lu, W.; Rettenmeier, E.; Paszek, M.; Yueh, M.-F.; Tukey, R. H.; Trottier, J.; Barbier, O.; Chen, S. Drug Metab. Dispos. 2017, 45, 748754.

(22) Lin, A.; Sved Skottvoll, F.; Rayner, S.; Pedersen-Bjergaard, S.; Sullivan, G.; Krauss, S.; Ray Wilson, S.; Harrison, S. Electrophoresis 2020, 41, 56-64.

(23) Pedersen-Bjergaard, S.; Rasmussen, K. E. J. Chromatogr. A 2006, 1109, 183-190.

(24) Drouin, N.; Kubáň, P.; Rudaz, S.; Pedersen-Bjergaard, S.; Schappler, J. TrAC, Trends Anal. Chem. 2019, 113, 357-363.

(25) Gjelstad, A.; Rasmussen, K. E.; Pedersen-Bjergaard, S. Anal. Bioanal. Chem. 2009, 393, 921-928.

(26) Eibak, L. E. E.; Rasmussen, K. E.; Øiestad, E. L.; PedersenBjergaard, S.; Gjelstad, A. Anal. Chim. Acta 2014, 828, 46-52.

(27) Drouin, N.; Mandscheff, J.-F.; Rudaz, S.; Schappler, J. Anal. Chem. 2017, 89, 6346-6350.

(28) Restan, M. S.; Pedersen, M. E.; Jensen, H.; Pedersen-Bjergaard, S. Anal. Chem. 2019, 91, 6702-6708.

(29) Petersen, N. J.; Jensen, H.; Hansen, S. H.; Foss, S. T.; Snakenborg, D.; Pedersen-Bjergaard, S. Microfluid. Nanofluid. 2010, 9, $881-888$.

(30) Hansen, F. A.; Sticker, D.; Kutter, J. P.; Petersen, N. J.; Pedersen-Bjergaard, S. Anal. Chem. 2018, 90, 9322-9329.

(31) Kamendulis, L. M.; Brzezinski, M. R.; Pindel, E. V.; Bosron, W. F.; Dean, R. A. J. Pharmacol. Exp. Ther. 1996, 279, 713.

(32) Rook, E. J.; Alwin, D. R. H.; Brink, W. v. d.; Ree, J. M. v.; Jos, H. B. Curr. Clin. Pharmacol. 2006, 1, 109-118.

(33) Maurer, H. H.; Sauer, C.; Theobald, D. S. Ther. Drug Monit. 2006, 28, 447-453.

(34) Ang, L. T.; Tan, A. K. Y.; Autio, M. I.; Goh, S. H.; Choo, S. H.; Lee, K. L.; Tan, J.; Pan, B.; Lee, J. J. H.; Lum, J. J.; Lim, C. Y. Y.; Yeo, I. K. X.; Wong, C. J. Y.; Liu, M.; Oh, J. L. L.; Chia, C. P. L.; Loh, C. H.; Chen, A.; Chen, Q.; Weissman, I. L.; et al. Cell Rep. 2018, 22, 2190-2205.

(35) Mathapati, S.; Siller, R.; Impellizzeri, A. A. R.; Lycke, M.; Vegheim, K.; Almaas, R.; Sullivan, G. J. Curr. Protoc. Stem Cell Biol. 2016, 38, 1G.6.1-1G.6.18.

(36) Siller, R.; Greenhough, S.; Naumovska, E.; Sullivan, G. J. Stem Cell Rep. 2015, 4, 939-952.

(37) Siller, R.; Naumovska, E.; Mathapati, S.; Lycke, M.; Greenhough, S.; Sullivan, G. J. Sci. Rep. 2016, 6, No. 37178.

(38) Siller, R.; Sullivan, G. J. Curr. Protoc. Stem Cell Biol. 2017, 43, 1G.7.1-1G.7.23

(39) Harrison, S. P.; Sillar, R.; Tanaka, Y.; Xiang, Y.; Patterson, B.; Kempf, H.; Melum, E.; Åsrud, K.; Chollet, M. E.; Andersen, E.; Sandset, P. M.; Baumgarten, S.; Bonanini, F.; Kurek, D.; Mathapati, 
S.; Almaas, R.; Sharma, K.; Wilson, S. R.; Skottvoll, F. S.; Boger, I. C.; et al. bioRxiv 2020, DOI: 10.1101/2020.12.02.406835.

(40) Hansen, F. A.; Jensen, H.; Pedersen-Bjergaard, S. Anal. Chem. 2020, 92, 5595-5603.

(41) Karinen, R.; Andersen, J. M.; Ripel, Å.; Hasvold, I.; Hopen, A. B.; Mørland, J.; Christophersen, A. S. J. Anal. Toxicol. 2009, 33, 345350 .

(42) Berg, H. S.; Seterdal, K. E.; Smetop, T.; Rozenvalds, R.; Brandtzaeg, O. K.; Vehus, T.; Lundanes, E.; Wilson, S. R. J. Chromatogr. A 2017, 1498, 111-119.

(43) Skottvoll, F. S.; Berg, H. E.; Bjørseth, K.; Lund, K.; Roos, N.; Bekhradnia, S.; Thiede, B.; Sandberg, C.; Vik-Mo, E. O.; RobergLarsen, H.; Nyström, B.; Lundanes, E.; Wilson, S. R. Future Sci. OA 2018, 5, No. FSO359.

(44) Pedersen-Bjergaard, S. Anal. Bioanal. Chem. 2019, 411, 16871693.

(45) Chan, S. D. H.; Toyoda, H.; Sanjeeviraman, J.; Souppe, A.; Iwamoto, M.; Wu, W.; Eto, D.; Tada, T.; Kumada, T.; Zhang, J.-P. Sci. Rep. 2020, 10, No. 7379.

(46) Ou, X.; Chen, P.; Huang, X.; Li, S.; Liu, B.-F. J. Sep. Sci. 2020, 43, 258-270.

(47) Cong, H.; Xu, X.; Yu, B.; Yuan, H.; Peng, Q.; Tian, C. J. Micromech. Microeng. 2015, 25, No. 053001.

(48) Fanali, S. Electrophoresis 2017, 38, 1822-1829.

(49) Yamini, Y.; Pourali, A.; Seidi, S.; Rezazadeh, M. Anal. Methods 2014, 6, 5554-5565.

(50) Rahimi, A.; Nojavan, S.; Tabani, H. J. Pharm. Biomed. Anal. 2020, 184, No. 113175.

(51) Ahmar, H.; Tabani, H.; Hossein Koruni, M.; Davarani, S. S. H.; Fakhari, A. R. Biosens. Bioelectron. 2014, 54, 189-194.

(52) Huang, C.; Seip, K. F.; Gjelstad, A.; Pedersen-Bjergaard, S. Anal. Chim. Acta 2016, 934, 80-87.

(53) Drouin, N.; Rudaz, S.; Schappler, J. J. Pharm. Biomed. Anal. 2018, 159, 53-59.

(54) Qian, Y.; Gilliland, T. K.; Markowitz, J. S. Chem. Biol. Interact. 2020, 316, No. 108914.

(55) Román-Hidalgo, C.; Martín-Valero, M. J.; Fernández-Torres, R.; Callejón-Mochón, M.; Bello-López, M. Á. Talanta 2017, 162, 3237.

(56) Wang, X.; Liang, Y.; Liu, L.; Shi, J.; Zhu, H.-J. Rapid Commun. Mass Spectrom. 2016, 30, 553-561.

(57) Prasad, B.; Bhatt, D. K.; Johnson, K.; Chapa, R.; Chu, X.; Salphati, L.; Xiao, G.; Lee, C.; Hop, C. E. C. A.; Mathias, A.; Lai, Y.; Liao, M.; Humphreys, W. G.; Kumer, S. C.; Unadkat, J. D. Drug Metab. Dispos. 2018, 46, 943.

(58) Wang, X.; Shi, J.; Zhu, H.-J. Proteomics 2019, 19, No. 1800288.

(59) Boberg, M.; Vrana, M.; Mehrotra, A.; Pearce, R. E.; Gaedigk, A.; Bhatt, D. K.; Leeder, J. S.; Prasad, B. Drug Metab. Dispos. 2017, 45, 216-223.

(60) Sato, Y.; Miyashita, A.; Iwatsubo, T.; Usui, T. Drug Metab. Dispos. 2012, 40, 1389-1396. 